\title{
Inflammation initiated by stressed organelles
}

Authors: Fabio Martinon ${ }^{1 *}$

\author{
Affiliations: \\ ${ }^{1}$ Departement of Biochemistry, University of Lausanne, Epalinges 1066, Switzerland. \\ *Correspondence to: \\ Fabio Martinon, \\ Departement of Biochemistry \\ University of Lausanne, \\ Ch. Des Boveresses 155 \\ Epalinges 1066, Switzerland \\ Phone: +41-21-692.5695 \\ Fax: +41-21-692.5705 \\ Email: Fabio.Martinon@unil.ch
}

Conflicts of interest statement: The author discloses no conflicts of interest.

Running title: stress pathways promoting inflammation

Keywords: Inflammasome, ER-stress, mitochondria, nuclear envelope

Words count : 3317 (main text abstract and legends)

Number of Figures: 3 


\begin{abstract}
:
Key cellular functions including those related to energy metabolism, organization of the genetic information or production of membrane-bound and secreted proteins are compartmentalized within organelles. Various stresses such as differentiation programs, viral and bacterial infections, perturbations in protein production, mechanical constraints, changes in the environment and nutriment accessibility can impact cellular homeostasis and organelle integrity. Perturbations of these cellular compartments trigger repair and adaptation programs aimed at restoring homeostasis. These events are often associated with low-grade inflammation also termed parainflammation. While the nature and mechanisms of danger-signals released by irremediably damaged cells are well understood, how transiently stressed cells trigger inflammation is still poorly understood. Emerging studies highlighted new mechanisms by which stress pathways promote inflammation. Cytosolic innate immune pathways are engaged by signals stemming from perturbed organelles such as the mitochondria, the endoplasmic reticulum (ER) or the nuclear envelope (NE). These observations indicate that these pathways function as guardians of cellular homeostasis and may contribute to disease in pathologies characterized by perturbations of cellular homoeostasis. Mitochondria stress, ER-stress or NE-stress are emerging as proinflammatory condition that contribute to human conditions and diseases.
\end{abstract}




\section{Introduction}

Innate immune systems have evolved to orchestrate immune responses in the context of infection and/ or injury. Sensors have been developed to detect pathogens while other detect stress. Early studies in plants have shown that some innate immune receptors including those related to the NOD-like receptors (NLRs) can function as guardian of cellular integrity [1]. These sensors organize surveillance mechanisms that detect perturbations of cellular physiology leading to the activation of immune responses. This system contributes to immune reactions aimed at fighting infection, but it also plays an important role in repairing damaged tissues and the restoration of cellular homeostasis [2]. The response resulting from these sensors is aimed at protecting the organism and sustaining tissue repair. It contributes to the removing of cellular debris originated by dead cells and promotes cell proliferation [3]. Inability to repair or eliminate the insult, as well as deregulation of these stress-sensing mechanisms, can trigger a low-grade underlining inflammation also termed parainflammation [4]. This response has been proposed to contribute to various conditions, diseases and pathologies including metabolic disorders, cancer and aging [5]. Perturbations of cellular integrity can also contribute to inflammation in rheumatic diseases. For example in gout, large crystals of monosodium urate trigger membrane destabilization and a process termed "frustrated phagocytosis" that promotes cellular perturbations causing the activation of the inflammasome.

Pathways and mechanisms monitoring the integrity of organelles such as the mitochondria, the endoplasmic reticulum (ER) and the nuclear envelope are emerging as central component of immune responses to cellular stress. In this review we will discuss recent advance identifying inflammatory signals stemming from these organelles, once stressed or damaged. 


\section{Stress signals}

In contrast to danger signals that are molecules and factors generally released by irremediably damaged cells, stress pathways and signals are produced by cells experiencing some sort of reversible loss of function. In this context the restoration of cellular function and homeostasis relies on an adaptation program that eliminates the insult and orchestrates the repair of the affected cellular compartment. As a result the inflammatory pathways originating form stressed organelle can be initiated by the active release of signals and factors as part of the adaptation response. Alternatively the release of these signals may directly result from the damage itself. Another major difference that distinguishes danger signals from stress signals, is the fact that stress signals can effectively trigger innate immune pathways directly from stressed cells in a cell-autonomous manner provided that these cells are intact and functioning.

\section{Mitochondria-stress mediated NLRP3 inflammasome activation}

Mitochondria are involved in wide-range of key cellular functions related to cellular metabolism and bioenergetics. These organelles function as metabolic hubs within the cell that can modify their physiology to meet cellular needs [6]. As part of the adaptation response, mitochondria can integrate intrinsic and extrinsic signals to adapt their function and number. Mitochondria can be targeted for destruction through mitophagy. This allows for example the elimination of damaged mitochondria. These organelles can also respond to stimuli and damage by actively releasing signals, including signals that modulate inflammatory pathways.

The importance of mitochondria in innate immunity is exemplified by the fact that several innate immune pathways are assembled on mitochondria that provide proper localization to their corresponding signalosomes. The assembly at the mitochondria and subsequent oligomerization of MAVS (mitochondrial antiviral signaling protein) also known as CARDIF (CARD adaptor 
inducing IFN- $\beta$ ) is essential for the engagement of type I interferon responses early following the detection of viral RNA within the cytosol of infected cells. Localization of the NLRP3 inflammasome at the mitochondria has also been reported to be a key event leading to its activation $[7,8]$.

Mitochondria are also participants in the initiation of innate immune signaling. Stressed mitochondria release inflammatory products that contribute to the engagement of inflammatory pathways. The best characterized one being the NLRP3 inflammasome (Figure 1).

NLRP3 is a scaffold protein that upon activation oligomerizes and recruits the adaptor ASC and the protease caspase-1. Following initial recruitment within the inflammasome, ASC can further assemble into high-molecular weight complexes by a process that resembles "prion-like" polymerization [9]. This engages most ASC molecules present within the cytosol of the cell into one active complex that recruits and promotes the activation of caspase-1. Inflammation is then initiated by the proteolytic maturation and of the cytokines IL-1 $\beta$ and IL-18.

Mitochondria are dynamic and continually change shape through the combined mechanisms of fission and fusion. Factor involved in mitochondrial dynamics have been found to impact NLRP3 activation. Decreased expression of the dynamin-related protein 1 (Drp1) triggers a defect in mitochondria fission that potentiates NLRP3 engagement [10]. In contrast, mitofusin-2 a positive regulator of mitochondria fusion has been shown to participate in NLRP3 activation. NLRP3 inflammasome activation can also be impacted by factors that regulate the removal of damaged mitochondria via mitophagy. Sestrin-2 has been proposed to decrease NLRP3 inflammasome assembly by promoting mitophagy [11]. Similarly, genetic defects in the autophagy machinery impacting on mitophagy have been found to contribute to inflammation in Crohn's disease [12]. 
Most described conditions and stimuli that trigger the NLRP3 inflammasome assembly and activation are characterized by perturbations of mitochondria homeostasis. It is likely that the release of mitochondria products function as signals leading to the engagement of NLRP3. The release of mitochondrial reactive oxygen species (mROS), and the release of mitochondrial DNA (mDNA) have been proposed to trigger NLRP3 activation [6, 13].

The release of mROS has been involved in autoinflammatory diseases. For example, in TNFR1associated periodic syndrome (TRAPS) patients, mutations within the TNFR1 have been shown to promote the generation of mROS [14]. This could explain the contribution of IL-1 $\beta$ and the inflammasome in this disease

The oxidized form of xanthine dehydrogenase, xanthine oxidase (XO) contribute to the formation of uric acid as well as mROS, Interestingly the inhibition of XO was shown to dampen inflammasome activation, suggesting that drugs used in gout to target $\mathrm{XO}$ and lower uric acid levels, may also impact mitochondria-triggered inflammation [15]. Whether these drugs could be of interest and repositioned in another autoinflammatory diseases such as TRAPS, is an hypothesis that remains to be tested.

The mechanisms by which mROS and other factors, possibly released by the mitochondria, activate the NLRP3 inflammasome are still not completely understood. One possibility is that mROS directly activates NEK7, a member of the family of mammalian NIMA-related kinases (NEK proteins). NEK7 directly interacts with NLRP3 and was proposed to be the possible ligand linking stress signals to NLRP3 activation [16-18] (Figure 1).

\section{ER-stress mediated inflammasome activation}


The endoplasmic reticulum (ER) is a continuous network of flattened sacs or tubes encased in membranes. Several metabolic pathways are compartmentalized within the ER. The synthesis, folding, maturation and transport of virtually all membrane and secreted proteins takes place in the ER. Hence, this organelle is the central compartment that produces most ligands and receptors involved in cell-cell communication. The ER harbors well-characterized sensors that control its capacity to meet the fluctuations in demand for protein synthesis and restore homeostasis if the organelle is overloaded or damaged [19]. ER-stress is a hallmark that has been associated with various rheumatic diseases including rheumatoid arthritis and spondyloarthritis (for review see [20]), however the mechanisms linking ER-perturbations and inflammation are still poorly understood.

Initial studies have shown that the inflammatory caspases, caspase-12 and the human homologue caspase-4 were activated in conditions of ER-stress [21]. While the roles of these proteases in the context of ER-stress are still unknown or controversial, these early observations indicated the possibility that ER-stress may contribute to inflammation-related pathways [22]. Several studies provided evidences that the NLRP3 inflammasome could be involved in caspase-1 activation in the context of ER-stress. It was shown that treatment of macrophages with compounds that disrupt ER function such as thapsigargin and tunicamycin trigger inflammasome-dependent IL$1 \beta$ secretion [23] (Figure 2). ER-stress was found to initiates an hepatic NLRP3 inflammasome activation that triggers inflammation-mediated liver injury in chronic liver diseases [24]. Two other studies have suggested that ER-stress may promote inflammasome activation by mediating the up-regulation of the NLRP3 binding protein TXNIP in insulin-producing $\beta$-cells $[25,26]$. How TXNIP may regulate NLRP3 upon ER-stress is still unclear and awaits further studies. 
The adaptation to ER-stress is mediated by several membrane-bound stress sensors that detect perturbations within the ER lumen. Among these sensors, IRE1, is particularly well studied [19]. In the context of microbial infection with the Brucella abortus vaccine strain RB51, it was shown that ER-stress mediated IRE1 activation engages NLRP3 at the mitochondria. This triggers an amplification-loop that augments the release of mitochondrial signals such as mROS further increasing NLRP3 activation [27]. These findings suggest that ER-stress may target the mitochondria to promote inflammasome activation.

The relevance of the IRE1-inflammasome connection was also described in a model of atherosclerosis. IRE1 function is mediated by its ribnonuclease activity. In macrophages, inhibitors targeting its ability to cleave RNA molecules reduce inflammasome activation mediated by hyperlipidemia-induced ER stress [28]. Moreover, in mice, IRE1 inhibitors decreased lipid-induced IL-1 $\beta$ and IL-18 production in the serum and reduced atherosclerotic plaque size [29].

\section{IRE1-mediated activation of XBP1 and RIG-I}

Activation of the innate immune receptors TLR2 and TLR4 by their respective ligands has been shown to activate the ER stress response proteins IRE1. This promotes the activation of the downstream active transcription factor $\mathrm{XBP} 1$ and was found to enhance the secretion of inflammatory cytokines such as IL-6 by macrophages [30]. These findings indicate that ERstress signaling pathways exacerbate the transcription of pro-inflammatory programs. Such

response could be particularly relevant in stressed tissues exposed to microbial products and could lead to the production of an hyper responsive TLR response.

ER-stress mediated IRE1 activation has also been linked to the activation of the RIG-I pathway

(Figure 2). RIG-I is a member of a family of cytosolic innate immune receptors termed RIG-I 
like receptors (RLRs). RLRs are $\mathrm{DExD} / \mathrm{H}$ box RNA helicases that detect viral RNA ligands or processed self-mRNA in the cytoplasm. Upon activation, these sensors activate transcriptional programs with a type I interferon and $\mathrm{NF \kappa B}$ signatures, thus promoting innate immunity and inflammation. Activation of IRE1 is involved in the degradation of specific cellular mRNA by a mechanism referred to as regulated IRE1-dependent decay (RIDD). This cleavage generates specific modifications within the RNA degradation products. In fact RIDD products are single stranded 5'-OH and 2'-3'-cyclic phosphate mRNA fragments. It was shown that these mRNAs are then detected by RIG-I, leading to the activation of a RIG-I dependent immune response. This was shown for example in the context of ER-stress pathways initiated by bacterial toxins such as Cholera and Shiga toxins. These microbial factors have been shown to elicit the IRE1RIG-I response in order to amplify immune responses [31]. This pathway also been involved in trichohepatoenteric syndrome, a systemic interferonopathy, associated with deficiency in TTC37 and SKIV2L [32]. The products of these two genes are cytosolic proteins involved in the degradation of cellular mRNA and may contribute to the elimination of cytosolic amounts of RIDD products produced by ER-stress responses. Therefore, inflammation in these patients is probably caused by SKIV2L (and possibly TTC37) deficient removal ER-stress mediated RIDD products [32].

\section{ER-stress mediated NOD1 and NOD2 activation}

ER-stress has also been involved in the activation of the innate immune sensors NOD1 and NOD2 [33]. These proteins are cytosolic members of the intracellular NOD-like receptor family that function to promote host defenses against bacteria through the induction of inflammation and immune responses [34]. NOD1 and NOD2 typically detect pathogen associated molecular patterns (PAMPs). NOD1 is activated by a motif present in many Gram-negative bacteria and 
certain Gram-positive bacteria, whereas NOD2 senses a conserved peptidoglycan product ubiquitously present in bacteria. A recent report showed that the ER-stress inducers thapsigargin and dithiothreitol trigger the production of the cytokine IL-6 in a manner that relies on both NOD1 and NOD2 [33]. In this study it was found that mice infected with Brucella abortus elicited a proinflammatory responses that was reduced by NODs deficiency or upon treatment with the ER-stress inhibitor TUDCA. Similarly the response was blunted upon treatment with the IRE1 inhibitor KIRA6 indicating that IRE1 may link ER-stress to NODs activation. Overall these findings suggest that NOD proteins drive inflammatory pathways engaged by ER-stress. However the mechanisms by which IRE1 may directly activate NODs, the signals detected by NODs during ER-stress and the physiological relevance of this response in other ER-stressed tissues and ER-stress associated pathologies remain to be investigated.

\section{NE-stress mediated activation of the AIM2 inflammasome}

The nuclear envelope (NE) is a highly regulated membrane barrier that physically separates the nucleus and its content from the cytoplasm [35]. The nuclear envelope inner and outer membranes are continuous with the ER. However, despite this continuity, both the NE and ER membranes are comprised of specific proteins that fulfill distinct functions and make the NE and ER distinct compartments.

Transient alterations of nuclear envelope homeostasis is characteristic of different pathologies, including viral infections, laminopathies and cancer [36, 37]. For example, infection with HIV can provoke nuclear envelope instability characterized by prominent lamina gaps as well as large blebs of herniating chromatin resulting in the exposure of nuclear material within the cytoplasm [38]. Transient nuclear rupture is also a hallmark of cultured fibroblasts from laminopathy patients [39]. These patients have inherited mutations that impair the maturation of lamin A, a 
structural component of the nuclear lamina. As a consequence, discontinuities of nuclear envelope was observed in hearts of mutant mice deleted for lysine 32 of lamin A [40] and in the liver of lamin A deficient animals [41]. Cancer cells also often exhibit changes in lamin A expression and nuclear morphology that resembles the phenotype observed in laminopathies, indicating that cancer cells may experience transient nuclear envelope rupturing [42, 43]. Importantly the NE envelope rupture is not a definitive event that results allways in the elimination of damaged cells. The NE is able to repair. This was observed in setups using pipettes to pass the NE for direct nuclear injections of DNA. It was also suggested from observations in the context of viral infections and laminopathies, however the mechanism involved in NE repair and the exact nature of the NE-stress response pathways are largely unknown [37, 39]. Recent work uncovered quality control mechanisms that cope with damage of nuclear envelope proteins and ensure the integrity of the nuclear envelope membranes [44]. This further demonstrates that similar the ER, the NE hosts adaptation programs that ensure its integrity. Importantly, the concept of NE-stress responses was demonstrated experimentally; cancer cells and immune cells forced to squeeze through tiny pores were found to undergo repeated rupturing the nuclear envelope that was followed by repair and restoration of cellular homeostasis $[45,46]$, further indicating that a NE response is a significant cellular phenomenon that is possibly relevant to physiological processes and several diseases.

Recently using a pharmacological perturbation of the nuclear envelope structure it was found that NE-stress and transient membrane rupture engages cytosolic innate immune receptors [47]. In this study the anti-viral drug nelfinavir was found to inhibit lamin A maturation causing alterations of nuclear shapes and transient exchange of material between the nucleus and the 
cytoplasm. This promotes the exposure in the cytoplasm of nuclear DNA, therefore triggering the activation of cytosolic sensors of DNA such as cGAS and AIM2 [47](Figure 3).

AIM2 harbors a DNA-binding region that is known to detect the cytosolic release of microbial double-stranded. This triggers AIM2 oligomerization and the assembly of an inflammasome harboring the adaptor ASC and the protease caspase-1. Transfection experiments have indicated that AIM2 detects dsDNA in a sequence-independent manner and from virtually any origin, including pathogen of any type and mammalian DNA [48]. AIM2 detection of self-DNA has been proposed to contribute to inflammation is diseases. For example, it was shown that AIM2dependent release of IL-1 $\beta$ in the skin may contribute to psoriasis [49].

Similarly to AIM2, the cGAS-STING pathway was found to be activated upon transient rupture of the nuclear envelope [47]. The ER-localized protein STING (stimulator of IFN genes) is essential for IFN- $\beta$ production in response to a variety of DNA stimuli of bacterial, viral, parasitic, or synthetic origin. A key signaling pathway upstream of STING involves the cGAMP synthase (cGAS). Cyclic-GMP-AMP (cGAMP) is produced by cGAS upon detection of DNA. This molecule, then, directly interacts with STING leading to the activation of proinflammatory transcriptional program related to a typical type I interferon gene signature.

These observations suggest that NE-stress and associated inflammatory pathways could contribute to conditions and disease associated with transient NE rupture such as laminopathies and cancers, however the physiological importance of this model remain to be tested.

\section{Conclusions}

The emerging concept that stress pathways can initiate inflammatory responses has open several new avenues of research. The mechanisms linking stress signals to the innate immune machinery are still poorly understood. Identifying these mechanisms will provide further insight on the 
evolutionary roots of this sophisticated system. In particular it would of interest to identify how the different stress responses co-operate to orchestrate various responses. It appears that signals stemming from the ER co-operate with the mitochondria to activate the NLRP3 inflammasome. It is likely that other organelles may co-operate. The NE may communicate with the RE to mount the NE stress-adaptation response and possibly to modulate inflammatory pathways. Finally, while organelles stress is a hallmark of several diseases and conditions, the precise role of the resulting stress-associated inflammation in these diseases is still poorly understood. Deciphering these connections will provide new therapeutic targets to modulate stress pathways and inflammation in diseases and conditions characterized by ER-stress and inflammation such as laminopathies, obesity, cancer and aging.

\section{Acknowledgments}

FM is supported by supported by a grant from the European Research Council (ERC) starting grant (281996) and a grant from the Swiss National Science Foundation (310030_173152).

\section{Figure legends}

\section{Figure 1 Mitochondria role in the activation of the NLRP3 inflammasome}

NLRP3 activating signals such as extracellular ATP or monosodium urate crystals signal via specific receptors or via interactions with the plasma membrane. This promotes a cellular response that includes potassium efflux through ion channels, and mitochondrial perturbations leading to the production and release of mitochondrial DNA and mitochondrial ROS (mROS) into the cytosol. mROS possibly in combination with other released factors engages NLRP3 
activating factors such as NEK7, thereby promoting inflammasome assembly. The adaptor ASC is recruited to the NLRP3 inflammasome and nucleates into prion-like filaments. ASC recruits Caspase-1 and oligomerizes off the ASC filaments leading to its activation. Active caspase-1 promotes the proteolytic cleavage and maturation of proIL- $1 \beta$ into the biologically active IL- $1 \beta$ hence, initiating an inflammatory cascade.

\section{Figure 2 Inflammatory pathways triggered by endoplasmic reticulum stress}

ER-stress triggers the activation of the endoribonuclease IRE1. This promotes the activation of the transcription factor $\mathrm{XBP} 1$ that can stimulate cytokine production and exacerbate inflammatory responses. IRE1 activation can also lead to the activation of the NLR proteins NOD1 and NOD2. This triggers the kinase RIPK2 that promotes the activation of the proinflammatory transcription factor NFKB. IRE1 can engage the degradation of a specific mRNA pool by a process called IRE1-dependent decay of mRNA (RIDD). RIDD degradation products are recognized by the RLR sensor RIG-I leading to the activation of a MAVS dependent proinflammatory response. Finally IRE1 activation has also been proposed to induce NLRP3 translocation to the mitochondria leading to mitochondrial damage and release of NLRP3 activating signals, thereby promoting inflammasome activation and IL-1 $\beta$ secretion.

\section{Figure 3 Inflammasome activation upon destabilization of the nuclear envelope}

Perturbations in the maturation of the Lamin A triggers transient alterations of NE structures and causes the transient exposure of self-DNA. Released DNA is detected by AIM2 leading to the assembly and activation of the AIM2 inflammasome.

\section{References:}


[1] Schneider DS. Plant immunity and film Noir: what gumshoe detectives can teach us about plant-pathogen interactions. Cell 2002;109(5):537-40.

[2] Kotas ME, Medzhitov R. Homeostasis, inflammation, and disease susceptibility. Cell 2015;160(5):816-27.

[3] Minutti CM, Knipper JA, Allen JE, Zaiss DM. Tissue-specific contribution of macrophages to wound healing. Semin Cell Dev Biol 2017;61:3-11.

[4] Medzhitov R. Origin and physiological roles of inflammation. Nature 2008;454(7203):428-35.

[5] Feldman N, Rotter-Maskowitz A, Okun E. DAMPs as mediators of sterile inflammation in aging-related pathologies. Ageing Res Rev 2015;24(Pt A):29-39.

[6] Weinberg SE, Sena LA, Chandel NS. Mitochondria in the regulation of innate and adaptive immunity. Immunity 2015;42(3):406-17.

[7] Kepp O, Galluzzi L, Kroemer G. Mitochondrial control of the NLRP3 inflammasome. Nature immunology 2011;12(3):199-200.

[8] Zhou R, Yazdi AS, Menu P, Tschopp J. A role for mitochondria in NLRP3 inflammasome activation. Nature 2011;469(7329):221-5.

[9] Cai X, Chen J, Xu H, Liu S, Jiang QX, Halfmann R, et al. Prion-like polymerization underlies signal transduction in antiviral immune defense and inflammasome activation. Cell 2014;156(6):1207-22.

[10] Park S, Won JH, Hwang I, Hong S, Lee HK, Yu JW. Defective mitochondrial fission augments NLRP3 inflammasome activation. Scientific reports 2015;5:15489.

[11] Kim MJ, Bae SH, Ryu JC, Kwon Y, Oh JH, Kwon J, et al. SESN2/sestrin2 suppresses sepsis by inducing mitophagy and inhibiting NLRP3 activation in macrophages. Autophagy 2016;12(8):1272-91.

[12] Saitoh T, Fujita N, Jang MH, Uematsu S, Yang BG, Satoh T, et al. Loss of the autophagy protein Atg16L1 enhances endotoxin-induced IL-1beta production. Nature 2008;456(7219):264-8.

[13] Shimada K, Crother TR, Karlin J, Dagvadorj J, Chiba N, Chen S, et al. Oxidized mitochondrial DNA activates the NLRP3 inflammasome during apoptosis. Immunity 2012;36(3):401-14.

[14] Bulua AC, Simon A, Maddipati R, Pelletier M, Park H, Kim KY, et al. Mitochondrial reactive oxygen species promote production of proinflammatory cytokines and are elevated in TNFR1-associated periodic syndrome (TRAPS). The Journal of experimental medicine 2011;208(3):519-33.

[15] Ives A, Nomura J, Martinon F, Roger T, LeRoy D, Miner JN, et al. Xanthine oxidoreductase regulates macrophage IL1beta secretion upon NLRP3 inflammasome activation. Nature communications 2015;6:6555.

[16] He Y, Zeng MY, Yang D, Motro B, Nunez G. NEK7 is an essential mediator of NLRP3 activation downstream of potassium efflux. Nature 2016;530(7590):354-7. 
[17] Schmid-Burgk JL, Chauhan D, Schmidt T, Ebert TS, Reinhardt J, Endl E, et al. A Genome-wide CRISPR (Clustered Regularly Interspaced Short Palindromic Repeats) Screen Identifies NEK7 as an Essential Component of NLRP3 Inflammasome Activation. The Journal of biological chemistry 2016;291(1):103-9.

[18] Shi H, Wang Y, Li X, Zhan X, Tang M, Fina M, et al. NLRP3 activation and mitosis are mutually exclusive events coordinated by NEK7, a new inflammasome component. Nature immunology 2016;17(3):250-8.

[19] Walter P, Ron D. The unfolded protein response: from stress pathway to homeostatic regulation. Science 2011;334(6059):1081-6.

[20] Navid F, Colbert RA. Causes and consequences of endoplasmic reticulum stress in rheumatic disease. Nature reviews Rheumatology 2017;13(1):25-40.

[21] Nakagawa T, Zhu H, Morishima N, Li E, Xu J, Yankner BA, et al. Caspase-12 mediates endoplasmic-reticulum-specific apoptosis and cytotoxicity by amyloid-beta. Nature 2000;403(6765):98-103.

[22] Martinon F, Tschopp J. Inflammatory caspases and inflammasomes: master switches of inflammation. Cell death and differentiation 2007;14(1):10-22.

[23] Menu P, Mayor A, Zhou R, Tardivel A, Ichijo H, Mori K, et al. ER stress activates the NLRP3 inflammasome via an UPR-independent pathway. Cell death \& disease 2012;3:e261.

[24] Lebeaupin C, Proics E, de Bieville CH, Rousseau D, Bonnafous S, Patouraux S, et al. ER stress induces NLRP3 inflammasome activation and hepatocyte death. Cell death \& disease 2015;6:e1879.

[25] Oslowski CM, Hara T, O'Sullivan-Murphy B, Kanekura K, Lu S, Hara M, et al. Thioredoxin-interacting protein mediates ER stress-induced beta cell death through initiation of the inflammasome. Cell metabolism 2012;16(2):265-73.

[26] Lerner AG, Upton JP, Praveen PV, Ghosh R, Nakagawa Y, Igbaria A, et al. IRE1alpha induces thioredoxin-interacting protein to activate the NLRP3 inflammasome and promote programmed cell death under irremediable ER stress. Cell metabolism 2012;16(2):250-64.

[27] Bronner DN, Abuaita BH, Chen X, Fitzgerald KA, Nunez G, He Y, et al. Endoplasmic Reticulum Stress Activates the Inflammasome via NLRP3- and Caspase-2-Driven Mitochondrial Damage. Immunity 2015;43(3):451-62.

[28] Robblee MM, Kim CC, Porter Abate J, Valdearcos M, Sandlund KL, Shenoy MK, et al. Saturated Fatty Acids Engage an IRE1alpha-Dependent Pathway to Activate the NLRP3 Inflammasome in Myeloid Cells. Cell reports 2016;14(11):2611-23.

[29] Tufanli O, Telkoparan Akillilar P, Acosta-Alvear D, Kocaturk B, Onat UI, Hamid SM, et al. Targeting IRE1 with small molecules counteracts progression of atherosclerosis. Proceedings of the National Academy of Sciences of the United States of America 2017;114(8):E1395-E404. 
[30] Martinon F, Chen X, Lee AH, Glimcher LH. TLR activation of the transcription factor $\mathrm{XBP} 1$ regulates innate immune responses in macrophages. Nature immunology 2010;11(5):411-8.

[31] Cho JA, Lee AH, Platzer B, Cross BC, Gardner BM, De Luca H, et al. The unfolded protein response element IRE1alpha senses bacterial proteins invading the ER to activate RIG-I and innate immune signaling. Cell host \& microbe 2013;13(5):558-69.

[32] Eckard SC, Rice GI, Fabre A, Badens C, Gray EE, Hartley JL, et al. The SKIV2L RNA exosome limits activation of the RIG-I-like receptors. Nature immunology 2014;15(9):839-45.

[33] Keestra-Gounder AM, Byndloss MX, Seyffert N, Young BM, Chavez-Arroyo A, Tsai AY, et al. NOD1 and NOD2 signalling links ER stress with inflammation. Nature 2016;532(7599):394-7.

[34] Caruso R, Nunez G. Innate Immunity: ER Stress Recruits NOD1 and NOD2 for Delivery of Inflammation. Current biology : CB 2016;26(12):R508-11.

[35] Hetzer MW. The nuclear envelope. Cold Spring Harbor perspectives in biology 2010;2(3):a000539.

[36] Davidson PM, Lammerding J. Broken nuclei--lamins, nuclear mechanics, and disease. Trends in cell biology 2014;24(4):247-56.

[37] Hatch E, Hetzer M. Breaching the nuclear envelope in development and disease. The Journal of cell biology 2014;205(2):133-41.

[38] de Noronha CM, Sherman MP, Lin HW, Cavrois MV, Moir RD, Goldman RD, et al. Dynamic disruptions in nuclear envelope architecture and integrity induced by HIV-1 Vpr. Science 2001;294(5544):1105-8.

[39] De Vos WH, Houben F, Kamps M, Malhas A, Verheyen F, Cox J, et al. Repetitive disruptions of the nuclear envelope invoke temporary loss of cellular compartmentalization in laminopathies. Human molecular genetics 2011;20(21):4175-86.

[40] Cattin ME, Bertrand AT, Schlossarek S, Le Bihan MC, Skov Jensen S, Neuber C, et al. Heterozygous LmnadelK32 mice develop dilated cardiomyopathy through a combined pathomechanism of haploinsufficiency and peptide toxicity. Human molecular genetics 2013;22(15):3152-64.

[41] Sullivan T, Escalante-Alcalde D, Bhatt H, Anver M, Bhat N, Nagashima K, et al. Loss of A-type lamin expression compromises nuclear envelope integrity leading to muscular dystrophy. The Journal of cell biology 1999;147(5):913-20.

[42] Prokocimer M, Margalit A, Gruenbaum Y. The nuclear lamina and its proposed roles in tumorigenesis: projection on the hematologic malignancies and future targeted therapy. Journal of structural biology 2006;155(2):351-60.

[43] Zink D, Fischer AH, Nickerson JA. Nuclear structure in cancer cells. Nature reviews Cancer 2004;4(9):677-87.

[44] Webster BM, Lusk CP. Border Safety: Quality Control at the Nuclear Envelope. Trends in cell biology 2016;26(1):29-39. 
[45] Denais CM, Gilbert RM, Isermann P, McGregor AL, te Lindert M, Weigelin B, et al. Nuclear envelope rupture and repair during cancer cell migration. Science 2016;352(6283):353-8.

[46] Raab M, Gentili M, de Belly H, Thiam HR, Vargas P, Jimenez AJ, et al. ESCRT III repairs nuclear envelope ruptures during cell migration to limit DNA damage and cell death. Science 2016;352(6283):359-62.

[47] Di Micco A, Frera G, Lugrin J, Jamilloux Y, Hsu ET, Tardivel A, et al. AIM2 inflammasome is activated by pharmacological disruption of nuclear envelope integrity. Proceedings of the National Academy of Sciences of the United States of America 2016;113(32):E4671-80.

[48] Rathinam VA, Jiang Z, Waggoner SN, Sharma S, Cole LE, Waggoner L, et al. The AIM2 inflammasome is essential for host defense against cytosolic bacteria and DNA viruses. Nature immunology 2010;11(5):395-402.

[49] Dombrowski Y, Koglin S, Schauber J. DNA-triggered AIM2 inflammasome activation in keratinocytes: Comment on Kopfnagel et al. Exp Dermatol. 2011. 20:1027-9. Experimental dermatology 2012;21(6):474-5; author reply 5-6. 


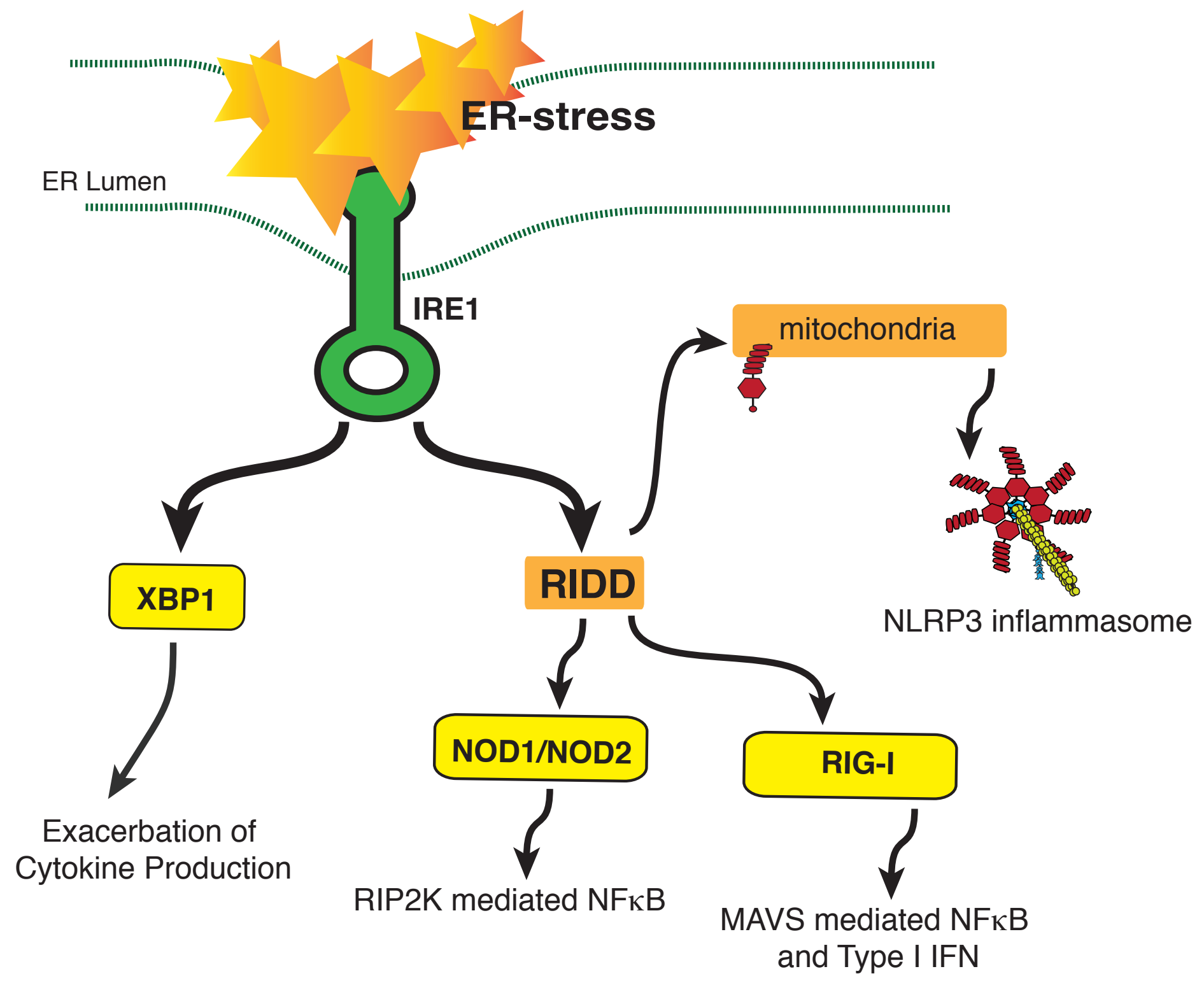

Figure 2 
Stress, mutations etc..

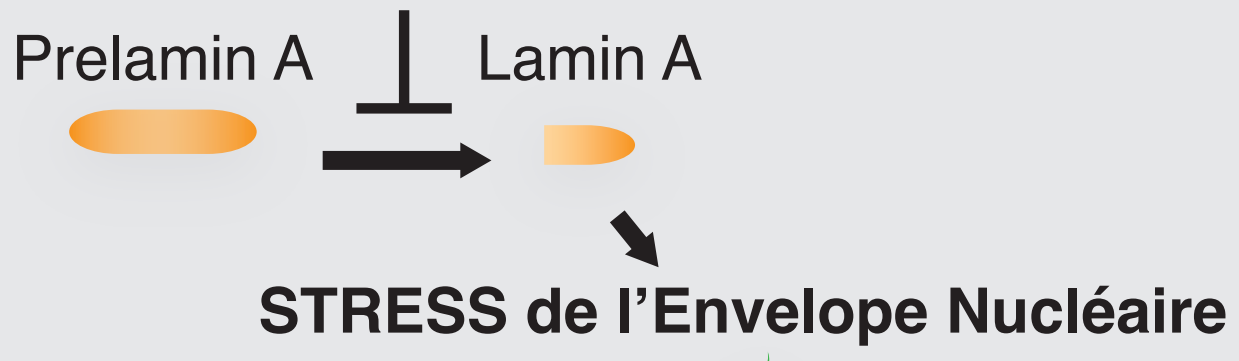

\section{Noyau}

Cytosol

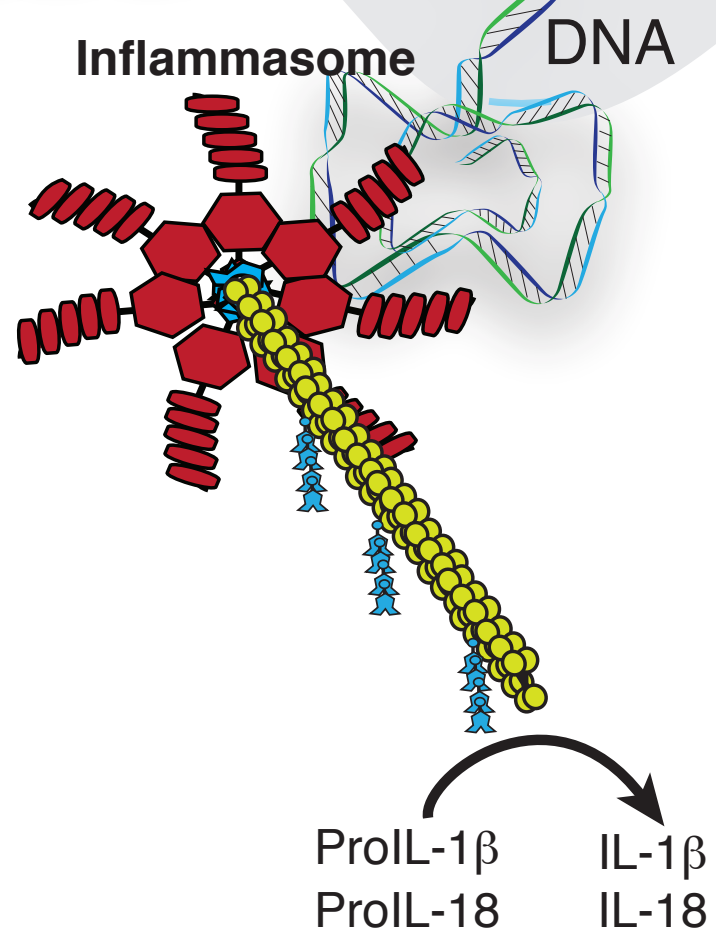

